\section{Effect of Autologous and Al- logeneic Platelet Rich Plasma on Viability, Proliferation and Chondrogenesis of Equine Bone Marrow Derived Mesen- chymal Stromal Cells}

\section{Lindsey Boone ${ }^{1 *}$, Jennifer Mumaw ${ }^{2}$, Merrilee Thoresen ${ }^{2}$ and John Peroni ${ }^{2}$}

${ }^{1}$ JT Vaughan Large Animal Teaching Hospital, Auburn University, Alabama, USA

${ }^{2}$ Department of Large Animal Medicine, University of Georgia, Georgia, USA

\begin{abstract}
Biological therapeutics are used in practice to treat musculoskeletal injuries of the equine athlete and optimization of these treatments for enhancement of the healing process is needed. Platelet Rich Plasma (PRP) and Bone Marrow Derived Mesenchymal Stromal Cells (BMSCs) are often used in combination, even though the source of these biological products may be from different donors. Bone marrow derived mesenchymal stem cells were obtained from 6 healthy adult horses, culture expanded, and characterized through tri-lineage differentiation. BMSCs were cultured for 48-72 hours in Dulbecco's Modified Eagle's Medium (DMEM) supplemented with either $25 \%$ or $50 \%$ (v/v) autologous or allogeneic PRP. Cellular viability was measured at 24 and 72 hours. Cellular proliferation was assessed at 48 hours. Chondrogenesis was quantified after 28 days in culture. Autologous and allogeneic PRP supplementation of the culture medium caused a dose dependent decrease in BMSC viability after 24 to 72 hours of culture. Both autologous and allogeneic PRP significantly decreased BMSC proliferation after 48 hours regardless of percent supplementation. BMSC chondrogenesis was significantly enhanced with exposure to PRP compared to control chondrogenic medium, regardless of whether the PRP was autologous or allogeneic. Exposure to PRP may significantly alter BMSC viability and proliferation, but appears to enhance BMSC chondrogenesis.
\end{abstract}

*Corresponding author: Lindsey Boone, JT Vaughan Large Animal Teaching Hospital, Auburn University, Alabama, USA, Tel: +1 3348448513; E-mail: Ihb002@auburn.edu

Citation: Boone L, Mumaw J, Thoresen M, Peroni J (2017) Effect of Autologous and Allogeneic Platelet Rich Plasma on Viability, Proliferation and Chondrogenesis of Equine Bone Marrow Derived Mesenchymal Stromal Cells. J Anim Res Vet Sci 1: 001

Received: August 01, 2017; Accepted: September 04, 2017; Published: September 18, 2017

Copyright: (C) 2017 Boone L, et al. This is an open-access article distributed under the terms of the Creative Commons Attribution License, which permits unrestricted use, distribution, and reproduction in any medium, provided the original author and source are credited.
Keywords: Chondrogenesis; Equine bone marrow; Mesenchymal stromal cell; Platelet rich plasma

\author{
Abbreviations \\ OA: Osteoarthritis \\ MSC: Mesenchymal Stromal Cell \\ MHC: Major Histocompatibility Complex \\ BMSC: Bone Marrow Derived Stromal Cell \\ PRP: Platelet Rich Plasma \\ GAG: Glycosaminoglycan \\ MDPI: Multidisciplinary Digital Publishing Institute \\ DOA: Directory of Open Access Journals \\ TLA: Three Letter Acronym \\ LD: Linear Dichroism
}

\section{Introduction}

Joint pain due to Osteoarthritis (OA) is one of the leading causes of reduced quality of life and loss of performance in people and animals [1-3]. Spontaneous and post-traumatic OA in horses has been regarded as a valuable translational model for human OA because the slow progression and the propensity for joint trauma in horses have allowed for meaningful pathophysiological comparisons to be made between horses and people [4-7].

In an attempt to restore articular homeostasis, clinicians treat arthritic joints in horses with a symptom modifying approach using systemic non-steroidal anti-inflammatory drugs and polysulfated proteoglycans and/or intra-articular cortico-steroids and viscosupplementing non-sulfated glycosaminoglycans such as hyaluronic acid [8].

As joint disease progresses, the development of cartilage deterioration eventually leads to persistent articular pain which is often ineffectively addressed by the above mentioned therapeutics. In an attempt to seek more effective alternatives for this frustrating disease, scientists and clinicians have been exploring the use of acellular and cell based biological therapeutics to enhance the repair of damaged cartilage $[9,10]$.

Mesenchymal Stromal Cells (MSCs), have been the subject of research inquiry because they are an easily obtainable, self-renewing cellular therapy for musculoskeletal repair $[10,11]$ MSCs have been shown to display disease modifying characteristics by preventing or slowing articular cartilage destruction $[12,13]$ and promoting articular cartilage defect repair through formation of hyaline-like reparative tissue [14-16]. To maximize efficiency of treatment, research efforts have been made to understand the implications of using donor allogeneic MSCs for cartilage repair strategies [17]. The use of banked donor cells offers attractive possibilities such as reduced cell propagation times of a few days compared to several weeks needed to harvest and culture autologous cells. Allogeneic cells may also be carefully characterized prior to clinical use to ensure that these cells possess the most desirable phenotypic and functional characteristics for the intended therapeutic purpose. Furthermore, although all reactivity concerns remain, evidence would suggest that allogeneic MSCs evoke minimal immune responses and therefore can survive transplantation in part due to a differential expression of Major Histocompatibility Complexes (MHC) on their cell surface [18-21]. 
The direct effects articular injection of MSCs to treat joint disease has been evaluated [22,23], however, when dealing with focal cartilage deficits, such as osteochondral trauma or developmental abnormalities, it is more desirable to implant MSCs specifically to the site of injury. Surgical implantation of MSCs requires co-delivery of these cells with a suitable matrix or scaffold to assist with cellular retention within the cartilage defect [24]. A recent study showed that cartilage repair of human osteoarthritic knees was improved with direct implantation of MSCs into cartilage defects compared to MSC injection into the joint alone [24]. As a result, it is appropriate to investigate the biological relationship of a cellular suspension of MSCs and a provisional matrix such as Platelet Rich Plasma (PRP) that can be activated upon delivery to enhance cellular retention into cartilage defects.

Platelet Rich Plasma is a blood concentrate that can play a pivotal role in wound repair because of its high concentration of growth factors and its ability to coagulate and form a provisional fibrinous scaffold [25]. Exposure of chondrocytes and MSCs to PRP has been shown to enhance cellular proliferation, viability, and the expression of genes important for repair or regeneration of the articular cartilage matrix [26-30]. Additionally, PRP decreases the release of inflammatory cytokines from chondrocytes and synoviocytes [31,32] and enhances synoviocyte hyaluronic acid production [33,34]. For these reasons, PRP has been suggested as a direct intra-articular therapeutic $[35,36]$ and as a bioscaffold for MSC implantation in articular cartilage defects [37].

The overall goal of this study was to further clarify the effects of combining PRP with MSCs in vitro. Very few studies have evaluated the effects of this combination on MSC viability, proliferation and function and to further complicate matters the literature reports a wide range of PRP preparation techniques, final composition, mode of activation, and volume of supplementation making it difficult to draw definitive conclusions on the biological interactions between MSCs and PRP [28,38-41]. As an example, low volumes of homologous PRP have been used as MSC culture media substitutes to minimize the introduction of xenogenic elements prior to the clinical use of stem cell preparations [42]. Conversely, studies have shown a dose dependent effect of high volumes of PRP on cellular proliferation in MSC cultures [43]. This contrasting evidence does not help clarify the appropriate combination to use for clinical application of PRP as a cellular suspension media for intra-articular administration or direct implantation of MSCs [40,43]. Regardless of the many unexplored details regarding PRP-MSC combination therapy, clinical data would suggest that the administration of MSCs suspended in PRP leads to clinical improvement in naturally occurring $[35,44]$ and in experimentally-induced [36] osteoarthritis. Furthermore, in a study involving 165 horses with naturally occurring arthritis of various joints, clinical scores improved following intra-articular administration of allogeneic peripheral blood MSCs suspended in allogeneic PRP [35].

These encouraging clinical reports combined with a lack of research investigating the combination of MSCs and PRP prompted this study in which our overall goal was to evaluate the outcome of culturing MSCs with autologous and allogeneic PRP. We hypothesized that MSCs supplemented in culture with autologous PRP would demonstrate greater viability, proliferation, and chondrogenic differentiation potential than MSCs cultured in allogeneic PRP.

\section{Results}

\section{BMSC characterization}

A subset of BMSCs from all of the six horses used in this study successfully exhibited osteogenic, chondrogenic and adipogenic differentiation potential. Compared to BMSCs treated with control media, plastic adherent cells exposed to differentiation media exhibited positive uptake of von Kossa and 7\% Oil-Red-O staining to confirm osteogenic and adipogenic differentiation. Chondrogenic differentiation was confirmed by the formation of micromass cell aggregates that stained positively for $1 \%$ alcian blue (Figure 1).

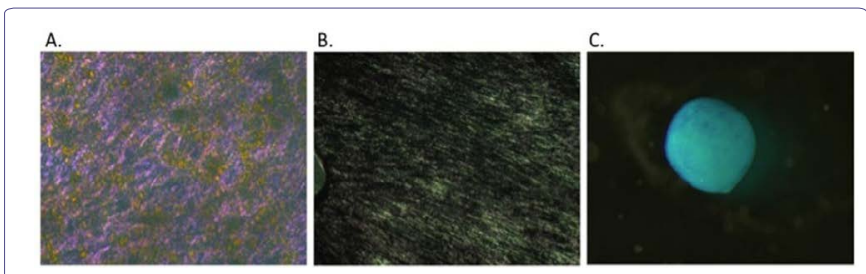

Figure 1: BMSC tri-lineage differentiation. Tri-lineage differentiation of one representative BMSC line. BMSCs stained positive for Oil Red O (A), Von Kossa (B), and Alcian blue (C).

\section{Platelet counts and growth factors.}

Concentrations of platelet rich preparations from each individual horse are displayed in table 1 . The mean platelet concentration was $2,668 \pm 209 \times 10^{3}$ cells $/ \mu$ l with a high of $3,185 \times$ $10^{3}$ cells $/ \mu \mathrm{l}$ and a low concentration of $2000 \times 10^{3} \mathrm{cells} / \mu \mathrm{l}$. We characterized growth factor concentrations from a subsample of our PRP preparations and growth factor concentration relative to platelet numbers was consistent across our preparations. Mean concentrations of growth factors relative to the platelet counts in the individual PRP samples were $9.4 \mathrm{ng} / \mathrm{ml} \pm 1.8 \mathrm{ng} /$ $\mathrm{ml}$ for TGF- $\beta 1,5.2 \mathrm{ng} / \mathrm{ml} \pm 2.2 \mathrm{ng} / \mathrm{ml}$ for PDGF-BB, and 14.6 $\mathrm{ng} / \mathrm{ml} \pm 16.0 \mathrm{ng} / \mathrm{ml}$ for VEGF-A.

\begin{tabular}{|c|c|}
\hline PRP Line & Platelet Count $\left(\times \mathbf{1 0}^{\mathbf{3}}\right.$ platelets $\left./ \boldsymbol{\mu l}\right)$ \\
\hline 1 & 3,185 \\
\hline 2 & 3,150 \\
\hline 3 & 2,235 \\
\hline 4 & 3,015 \\
\hline 5 & 2,000 \\
\hline 6 & 2,420 \\
\hline
\end{tabular}

Table 1: Platelet counts. Platelet counts $(\mathrm{cells} / \mu \mathrm{l})$ for each individual PRP.

\section{BMSC viability}

Compared to standard culture media, supplementation of BMSC cultures with $25 \%$ PRP significantly increased BMSC viability after 24 hours followed by a decline in viability at 72 hours regardless of the PRP source. Conversely, supplementing BMSC cultures with 50\% PRP significantly decreased BMSC viability at 24 and 72 hours regardless of the PRP source (Figure 2). 


\section{Cellular Proliferation via EdU DNA incorporation}

There was a significant decrease in cellular proliferation of BMSCs cultured in all PRP preparations compared to BMSCs cultured in media alone regardless of the source of the PRP (autologous or allogeneic). No significant differences between PRP preparations were found, but there was a trend for higher PRP concentrations to decrease the quantification of EDU suggesting a more pronounced decline in proliferation at the $50 \%$ PRP concentration (Figure 3).

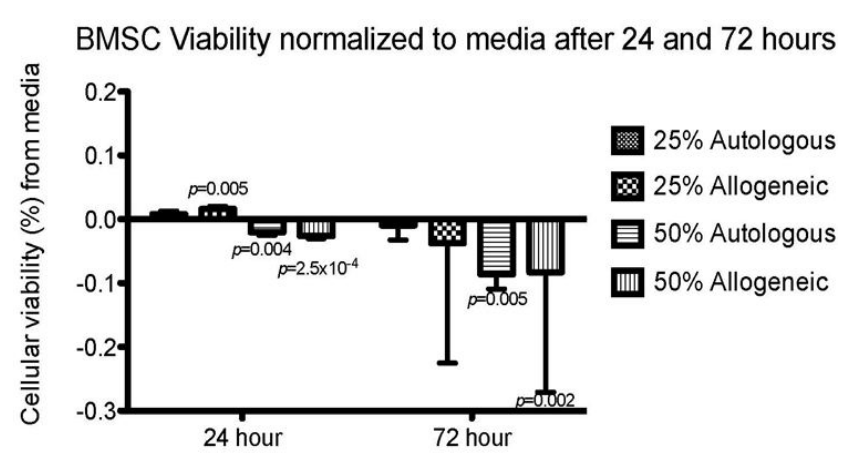

Figure 2: BMSC viability after 24 and 72 hours of culture. Mean percent of live BMSCs cultured for 24 hours in $25 \%$ autologous PRP $(n=6$ PRP lines tested), 25\% allogeneic PRP ( $\mathrm{n}=12$ PRP lines tested, 1 BMSC line exposed to 2 different allogeneic PRP sources) $50 \%$ autologous PRP ( $n=6$ PRP lines tested), and 50\% allogeneic PRP ( $n=12$ PRP lines tested, 1 BMSC line exposed to 2 different allogeneic PRP sources), 25\% allogeneic PRP, 50\% autologous PRP, and 50\% allogeneic PRP subtracted from mean percent of live BMSCs cultured in control medium (Baseline 0.0).

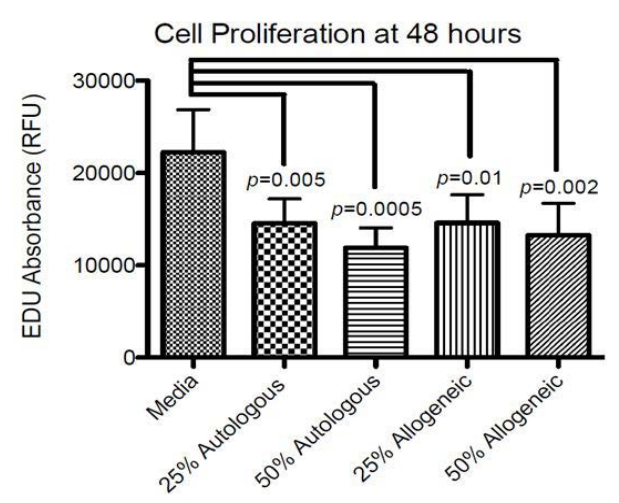

Figure 3: Cellular proliferation after 48 hours in culture. Mean absorbance (RFu) for Edu for BMSCs cultured for 48 hours. Significant differences $(\mathrm{p}<0.05)$ from media are displayed. No significant differences between treatment groups were identified. Media, 25\% autologous PRP, and 50\% autologous PRP groups contained data from the 6 BMSC lines ( $\mathrm{n}=6)$. Data for the $25 \%$ and $50 \%$ allogeneic groups was generated following exposure of 6 BMSC lines to 6 different PRP lines, but each BMSC line was exposed to 2 different allogeneic sources of PRP $(n=12)$

\section{Chondrogenesis}

There were no differences in the quantity of viable cells contained within chondrogenic pellets cultured in control chondrogenic medium and all PRP sources as determined by neutral red staining.

All PRP preparations enhanced GAG production compared to control chondrogenic medium and GAG production was higher with the higher concentrations of PRP (50\%). When GAG production was evaluated in relation to the percentage of viable BMSC, all PRP conditions produced significantly higher GAG per BMSC compared to chondrogenic medium, but no differences were detected between treatment groups (Figure 4).

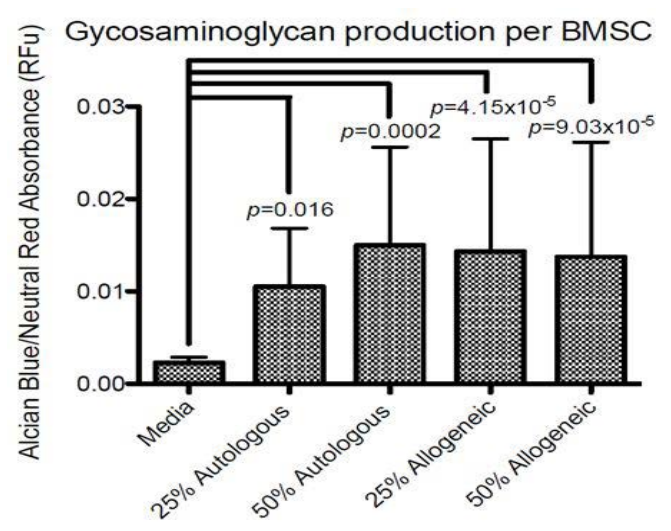

Figure 4: Glycosaminoglycan content per viable BMSC. Ratio of alcian blue absorbance (glycosaminoglycan content) to neutral red absorbance (cell number) for BMSC micromass pellets cultured for 28 days in $25 \%$ autologous PRP $(n=6$ PRP lines tested), $25 \%$ allogeneic PRP $(n=12$ PRP lines tested, 1 BMSC line exposed to 2 different allogeneic PRP sources) $50 \%$ autologous PRP $(\mathrm{n}=6$ PRP lines tested), and 50\% allogeneic PRP ( $=12$ PRP lines tested, 1 BMSC line exposed to 2 different allogeneic PRP sources). Results that are significantly different $(\mathrm{p}<0.05)$ from media are displayed as $p$ values overlying the treatment group from which a significant difference was seen.

No significant differences between treatment groups were identified.

\section{Discussion}

Platelet rich plasma is an easily obtainable blood derived biological product that serves as a scaffold and a source of growth factors shown to variably sustain or enhance the cellular proliferation, migration, and biological activity of progenitor cells from various tissue sources and species [45]. In this study we report a dose dependent effect of PRP on BMSC viability and proliferation, with increasing concentration of media supplementation of PRP causing reduced BMSC viability and proliferation. Contrary to our hypothesis, the PRP source (autologous vs. allogeneic) had no significant effect on cellular viability and proliferation. Interestingly and despite an overall decline in viability and proliferative capacity, chondrogenic differentiation of BMSC was enhanced by supplementing differentiation media with PRP irrespectively of the source or concentration.

The effect of PRP on the biological activity of various cell types has been the subject of a number of research papers $[28,38,39,41,46]$. Very few of these papers, however, have considered investigating if PRP preparations autologous or allogeneic to the MSC source would impact the biology and development of MSCs [47]. In this study, the viability, proliferation, and the chondrogenic differentiation potential of equine BMSCs were similar regardless of exposure of the cells to autologous or allogeneic PRP. It is worth noting that incubation of MSCs in allogenic serum has been shown to significantly enhance MSC cytotoxicity via the activation of complement and formation of membrane attack complexes compared to incubation of MSCs in autologous serum [48]. Plasma retains components of the complement system along with naturally occurring allo-antibodies and platelet micro particles/membranes which contain complex surface antigens 
that could be recognized by the recipient immune system. It was beyond the scope of this study to address the potential immunogenicity of BMSCs following exposure to allogeneic PRP, however, mixed leukocyte reaction studies or co-incubation of media obtained from BMSCs exposed to allo-PRP could be investigated to further elucidate this important aspect of BMSC biology.

Numerous studies address cellular proliferation [43,49-53], but few studies address cellular viability following supplementation with platelet products in vitro $[43,54]$. These studies were primarily conducted to evaluate platelet products as an alternative growth supplement for MSCs, to address the concern that fetal bovine or calf serum contain xenogenic proteins that could enhance the immune response directed toward MSCs, leading to decreased therapeutic efficacy. Most of the aforementioned studies, evaluated low volumes $(<20 \%)$ of media supplementation with platelet derived products but little is known about the effects of higher concentrations of PRP on MSCs. Higher media supplementation in vitro would more closely mimic a clinical scenario in which PRP and MSCs are admixed immediately prior to injection into a joint or cartilage defect $[43,49,53]$.

In the present study the platelet counts achieved with our centrifugation technique (average of 14.3-fold increase from a baseline platelet count) are higher than those investigated in the literature (2-10 fold increase from baseline). The ideal concentration of platelets in a therapeutic PRP preparation is unknown, but ranges from 3 to 6 fold higher than peripheral blood are recommended in the literature for various clinical applications. Growth factor concentrations correlate to platelet numbers hence the desire to maximize platelet concentration in our clinical practice. The relatively high platelet counts may have influenced the results our study, however growth factor analysis characterizing our PRP preps showed very similar average concentrations of growth factors relative to platelet numbers. Nevertheless, our individual platelet concentrations differed and this may have played a role in the variability of our results. At the same time, we chose not to standardize platelet numbers in order to mimic as closely as possible the clinical scenario in which MSCs would be admixed with a preparation containing platelet concentrations that vary dependent on the donor and processing technique. PRP is a biological product that is subject to great variability due to differences in collection and processing as well as differences within and between subjects. This variability in PRP preparation is a major limitation of this study and makes it difficult to draw definitive conclusions about the effect of PRP on BMSCs when comparing to other studies.

After a 24-hour exposure to $25 \%$ PRP, BMSC viability was comparable (autologous) or slightly improved (allogeneic) to that seen with control medium whereas exposure to $50 \%$ PRP significantly reduced BMSC viability. This decline in cellular viability with increasing concentration of platelet derived product is consistent with the findings of Russell et al., [43] in which umbilical cord blood derived MSC viability declined with $>30 \%$ supplementation of culture media with pooled allogeneic platelet lysate. Furthermore, Garvican et al., [53] showed significant reduction in BMSC viability following suspension in $100 \%$ allogeneic PRP and storage at $4-8^{\circ} \mathrm{C}$. The reason for this reduced cellular viability following PRP exposure and/or suspension is unknown. Possible mechanisms include immune mediated cytotoxicity via the complement pathway as mentioned above or cytotoxicity associated with an acidic $\mathrm{pH}$. In our study, BMSC viability may have been conditioned by the relatively acidic $\mathrm{pH}$ resulting from exposure to high concentration of platelets especially at the $50 \%$ concentration. There is evidence to suggest that buffering PRP prior to therapeutic application may enhance cellular viability prior to therapeutic application [27].

We report a significant decrease in cellular proliferation at $25 \%$ and 50\% PRP supplementation compared to media alone. This is in contrast, to a study by Del Bue et al., [49] which showed a dose dependent increase in adipose derived MSC proliferation up to $50 \%$ supplementation with platelet lysate and a study by Russell et al., [43] that showed no significant differences in umbilical cord blood derived MSC proliferation between the same volume of FBS and platelet lysate supplementation up to $30 \%$. In the study by Russell, there was dose dependent increase in cellular proliferation with a trend for platelet lysate to have greater proliferation rates compared to supplementation with the same volume of FBS, but following supplementation of $>30 \%$ platelet lysate umbilical cord derived MSC proliferation significantly declined while umbilical cord derived MSC proliferation continued to increase with increasing volume of supplementation. In the current study, BMSC proliferation was significantly decreased following supplementation with $25 \%$ and $50 \%$ PRP compared to standard culture media supplementation of $10 \%$ FBS. The trend for increasing PRP concentrations to cause reduction in BMSC proliferation and this is consistent with the findings of Russell et al., [43] in which supplementation with $>30 \%$ platelet lysate resulted in a dose dependent decline in umbilical cord derived MSC viability. Reasons for the initial dose-dependent increase followed by a rapid and steep decline in MSC proliferation following exposure to platelet products is unknown, but could be related to the platelet concentration and/ or protein (growth factor) composition of the platelet product. Additionally, direct comparison of platelet derived products is difficult given the varying cellular compositions that could also contribute to differences in MSC response, with platelet lysate being acellular and PRP containing white and red blood and platelet cellular fragments.

In the present study, direct contact of BMSCs with the PRP clot was not allowed to facilitate data measurement of the BMSCs. When PRP is activated it forms a gel-like matrix that incorporates platelet fragments, white blood cells, and red blood cells which could have contributed to the cellular viability and/or proliferation data in our experiment. Therefore, the PRP was activated within the culture system, but physically separated using a co-culture system and removed prior to performing viability and proliferation assays. The majority of studies that have evaluated the effects of platelet products on MSC viability, proliferation, or chondrogenesis use a PRP releasate or platelet lysate which contains released factors from activated platelets $[38,39,40,43,46]$. Creeper et al., [47] evaluated the effects of various concentrations of activated autologous and allogeneic PRP in direct contact during culture of gingival fibroblasts. No differences between autologous and allogeneic preparations on gingival fibroblast function were found [47]. Inhibition of direct contact between BMSCs and PRP in this study is a major limitation. However, the PRP clot was activated and remained within the culture system to ensure that the BMSCs were exposed to residual factors secreted from the PRP clot that may not be included in PRP releasate or platelet lysate in an attempt to mimic the clinical setting as best possible.

Platelet rich plasma had a significant effect on chondrogenic differentiation of BMSCs in comparison to chondrogenic medium alone, however a dose-dependent effect was not observed. The literature has shown that chondrogenesis of MSCs can be maintained or enhanced after supplementing culture medium with $5-10 \%$ platelet derived products [27,51,54-58]. The higher concentrations of PRP that we used in this study, however, are rarely evaluated in the literature. 
In the current study, 25\% and 50\% PRP concentrations were shown to enhance GAG production of differentiated BMSCs over medium alone. These higher supplementation volumes more closely reflect the clinical reality in which BMSCs are implanted in cartilage defects suspended in 100\% PRP suggesting that, PRP and BMSC combinations should be further evaluated for the purpose of cartilage defect delivery.

Measurement of GAG production of MSCs within micro mass pellet culture is a commonly used method to confirm chondrogenesis. Negatively charged GAGs are attached to the aggrecan molecules within the cartilage matrix and are responsible for cartilages compressive strength. Demonstration of type II collagen and GAG production within micro mass pellet culture and/or cartilage defect suggest the formation of a more hyaline-like tissue matrix resembling articular cartilage not fibro cartilage. PRP has been used as a cellular suspension for delivery into articular cartilage defects due to its important biological activity and its ability to form a provisional scaffold/matix within the defect site. Goodrich et al., [59] showed that equine BMSCs suspended in platelet-enriched fibrin and implanted in experimentally induced chondral defects supported formation of hyaline-like repair tissue. However, platelet-enriched fibrin outperformed BMSCs in repair of the chondral defects and ectopic bone formation was found in the repair tissue of some of the defects in which BMSCs and platelet enriched fibrin were used to fill the defect [59]. This is in contrast to our findings in which PRP enhanced chondrogenesis, though its effects of BMSC osteogenesis were not evaluated. This further supports the need for evaluating the role of MSCs and more clinically relevant concentrations of PRP both in vitro and in vivo.

Our findings indicate that there is no difference in viability, proliferation, or chondrogenesis of BMSCs treated with either $25 \%$ or $50 \%$ autologous or allogeneic PRP. Higher supplementation of PRP $(50 \%)$ reduces BMSC viability and proliferation, but enhances chondrogenic differentiation. Suspension of equine BMSCs in 25-50\% autologous or allogeneic PRP for injection may cause an acute decrease in cellular viability and cellular proliferation with enhancement of chondrogenesis. Although a direct extrapolation of our results to the clinical setting in which BMSCs may be mixed or re-suspended in nearly $50-100 \%$ PRP may not be accurate, our data raises the concern that BMSCs viability may deserve further scrutiny when these combinations are used.

\section{Materials and Methods}

\section{Animals}

Bone marrow for the proliferation of MSCs and blood for the production of PRP were aseptically harvested on separate dates from 6 healthy adult horses aged 8-14 years old. All procedures involving collection of samples for this study were done with the approval of the Animal Care and Use Committee at The University of Georgia.

\section{Isolation of BMSCs}

Bone marrow was aseptically harvested from the sternum of six healthy adult horses using an 8-gaugeX 4 in Jamshidi bone marrow biopsy needle. Approximately $20 \mathrm{cc}$ of bone marrow were drawn from either the $4^{\text {th }}$ or $5^{\text {th }}$ sternebrae into $2-35 \mathrm{cc}$ syringes containing 2500 units of heparin sulfate ${ }^{b}$ in each syringe. BMSCs were obtained using a plate adherency culture method. Cells were cultured in defined culture medium consisting of Dulbecco's Modified Eagles Medium with $4.5 \mathrm{~g} / \mathrm{L}$ glucose and sodium pyruvate without L-Glutamine ${ }^{\mathrm{c}}$ supplemented with $10 \%$ fetal bovine serum ${ }^{\mathrm{d}}, 4 \mathrm{mM}$ L-Glutamine and $50 \mu \mathrm{g} / \mathrm{ml}$ Streptomycin, $50 \mathrm{U} / \mathrm{ml}$ Penicillin ${ }^{\mathrm{f}}$ under standard cell culture conditions $\left(37^{\circ} \mathrm{C}\right.$ and $\left.5 \% \mathrm{CO}_{2}\right)$. Initial BMSC colonies were manually dissociated to allow re-distribution prior to trypsinization. Once the cells reached $70-80 \%$ confluency, they were harvested with $0.05 \%$ trypsin-EDTA ${ }^{g}$ and reseeded at $5000 \mathrm{ccells} / \mathrm{cm}^{2}$ for further expansion.

\section{BMSC characterization}

For osteogenic and adipogenic differentiation, 36,000 MSCs per $\mathrm{cm} 2$ from each of the 6 lines were plated in $35 \mathrm{~mm}$ plates. The cells were differentiated using Hyclone Advance STEM osteogenic differentiation medium $^{\mathrm{h}}$ with $50 \mu \mathrm{g} / \mathrm{ml}$ Streptomycin, $50 \mathrm{U} / \mathrm{ml}$ Penicillin, and Hyclone Advance STEM adipogenic differentiation medium ${ }^{i}$ with $50 \mu \mathrm{g} / \mathrm{ml}$ Streptomycin, $50 \mathrm{U} / \mathrm{ml}$ Penicillin respectively. The medium was changed every third day for 28 days. For osteogenic differentiation, cells were stained with Von Kossa ${ }^{j}$ and for adipogenic differentiation cells were stained with $7 \% \mathrm{O}^{\mathrm{k}}$. Chondrogenesis was performed using micromass pellets formed in $15 \mathrm{ml}$ polypropylene tubes. $1 \times 10^{6}$ cells were pelleted at $400 \mathrm{~g}$ and Advance STEM chondrogenic differentiation medium 1 was changed every third day for 28 days. Micro masses were stained with $1 \%$ alcian blue ${ }^{\mathrm{m}}$.

\section{Platelet rich plasma processing}

Approximately 1 liter of blood was aseptically harvested from the left jugular vein of the same 6 adult horses from which BMSCs lines were developed and collected in 2 blood collection bags containing $63 \mathrm{mls}$ of citrate phosphate dextrose adenine solution (CPDA-1 $)^{\mathrm{n}}$. The blood was subsequently processed aseptically under a direct laminar flow hood using standard cellular processing techniques. The blood was centrifuged at $1800 \mathrm{rpm}$ at $4^{\circ} \mathrm{C}$ for 10 minutes without automated deceleration. Plasma above the buffy coat was harvested and then centrifuged at $2400 \mathrm{rpm}$ at $4^{\circ} \mathrm{C}$ for 5 minutes with automated deceleration. The supernatant was decanted and then centrifuged at $3300 \mathrm{rpm}$ at $4^{\circ} \mathrm{C}$ for 5 minutes with automated deceleration. The platelet poor supernatant was decanted and discarded. The cellular pellets from the second and third centrifugation cycles were re-suspended in the remaining plasma after decantation and combined to produce PRP. A $200 \mu \mathrm{l}$ sample was obtained from the PRP to perform an automated platelet count. This method of PRP processing yielded approximately 10-15 mls of PRP from 1 liter of blood. To allow for analysis, BMSCs were kept separate from the PRP through the use of trans-well inserts with a $0.04 \mu \mathrm{M}$ pore size. BMSC groups were organized so that each of the 6 BMSC lines were exposed to autologous PRP and two allogeneic PRP samples. Treatment groups were as follows: defined culture medium, defined culture medium with $25 \%$ autologous PRP, defined culture medium with $50 \%$ autologous PRP, defined culture medium with $25 \%$ allogeneic PRP, and defined culture medium with $50 \%$ allogeneic PRP. The PRP was activated by the addition of $10 \% \mathrm{CaCl}_{2}$ to each of the trans-well inserts. PRP was activated to more closely reflect the clinical scenario for filling of a articular cartilage defect where MSCs and PRP would be injected together with an activating agent to allow clot formation of the PRP within the defect. Growth factor release from platelets is influenced by activation, therefore it was important to resemble this clinical scenario as closely as possible in vitro. However, direct contact with the PRP clot was not allowed to facilitate diffusion of reagents for cellular assays and facilitate data measurement.

PRP aliquots from each horse were analyzed using immuno assays 
(Quantikine Immunoassay, R\&D Systems, MN, USA) for platelet derived growth factor-BB (PDGF-BB) and transforming growth factor- $\beta 1$ (TGF- $\beta 1$ ) and immunoassay (Vet Set, Kingfisher, MN, USA) for Vascular Endothelial Growth Factor-A (VEGF-A) following the manufacturer's instructions.

\section{Proliferation assay}

BMSCs were plated at 3500 cells $/ \mathrm{cm} 2$. Proliferation of BMSCs in the presence of autologous or allogeneic PRP was assessed using the Clik-iT® EdU Microplate Assay ${ }^{\circ}$. 5-ethynyl-2'deoxyuridine (Edu) was added at a concentration of $10 \mu \mathrm{M}$ to the medium of all treatment groups and cells were cultured for 48 hours in the presence of Edu. Six hours prior to initiating the Clik-iT ${ }^{\circledR}$ EdU microplate assay the medium was removed from the plates and $150 \mu \mathrm{l}$ defined medium containing type IV collagenase $(1 \mathrm{mg} / \mathrm{mL})^{\mathrm{p}}$ and $50 \mu 1$ of $0.05 \%$ trypsin-EDTA were added to each well of the 96 well plate to allow dissolution of cells from the protein matrix that eluded from the trans well. The plates were then centrifuged and the assay was performed according to package insert instructions with the addition of centrifugation between steps. The plate was analyzed using a microplate reader set to read at 495 with correction at 519 .

\section{Viability assay}

BMSCs were plated at 3500 cells $/ \mathrm{cm}^{2}$. Viability of BMSCs was assessed using the LIVE/DEAD ${ }^{\circledR}$ viability/cytotoxicity kit for mammalian cells q after 24 and 72-hour incubation in autologous or allogeneic PRP. DNA quantification was performed with bis-Benzimide H33342 tri-hydrochlorider. Six hours prior to performing the assay cells were separated from the protein matrix as described above. The cells were then centrifuged and the assay was performed according to package insert instructions. The plate was then assessed for absorbance at 528 with correction set at $617 \mathrm{~nm}$ on a microplate reader.

\section{Chondrogenic assay}

BMSCs were seeded at density of 200,000 BMSCs/well in 96 well "V" bottom plates ${ }^{\mathrm{s}}$ for chondrogenesis. The plates were centrifuged at $400 \mathrm{~g}$ for $10 \mathrm{~min}$ to form micro mass pellets. 12 hours later the micro masses were transferred to trans well plates for an additional 24 hours prior to exposure to PRP and chondrogneic differentiation medium supplemented with $50 \mu \mathrm{g} / \mathrm{ml}$ Streptomycin and $50 \mathrm{U} / \mathrm{ml}$ Penicillin. Treatment groups were as follows: chondrogenic medium, chondrogenic medium supplemented with $25 \%$ activated autologous PRP, chondrogenic medium containing 50\% activated autologous PRP, chondrogenic medium containing $25 \%$ activated allogeneic PRP, and chondrogenic medium containing 50\% activated allogeneic PRP. 50\% medium changes were performed every other day. After 28 days in culture the transwell insert was removed and the micromass pellet was harvested from the protein matrix encasing the micromass pellet on the bottom of the insert and transferred to the correspondent well of the plate. The micromass pellets were rinsed 3 times with PBS and then fixed with $100 \%$ methanol for 10 minutes at $-20^{\circ} \mathrm{C}$. A $0.2 \%$ Alcian Blue $8 \mathrm{GX}$ in $0.1 \mathrm{M} \mathrm{HCl}$ solution was applied to the micromass pellets for 2 hours at room temperature. The pellets were then washed three times with PBS and alcian blue stain that had been taken up by the pellets was extracted by exposing the pellets to $6 \mathrm{M}$ guanidine $/ \mathrm{HCl}^{\mathrm{t}}$ overnight at room temperature. The optical density of the extracted alcian blue was measured at $650 \mathrm{~nm}$ on a micro plate reader. The quantity of viable cells within the pellets was measured by staining the pellets in parallel with Neutral Red ${ }^{u}$. The detection of neutral red content was measured at an optical density of $550 \mathrm{~nm}$.
Using this method cell viability and chondrogenesis were simultaneously quantified. The total GAG/cell content was measured by dividing the fluorescence alcian blue by the fluorescence of neutral red for each micro mass pellet.

\section{Statistical Analysis}

All analyses were performed using SAS V 9.2 ${ }^{\mathrm{v}}$. Control medium values were averaged over the three replicates for proliferation and chondrogenesis data to obtain a single control value for each horse. Control values were subtracted from viability, proliferation and chondrogenesis data prior to analysis to obtain change from control values for analysis.

A repeated measures model that recognized multiple observations as belonging to the same horse was used to test for differences in viability, proliferation and chondrogenesis change from control values between groups and PRP lines. The full model included fixed factors for group, PRP line, and an interaction effect of group and PRP line as well as a random factor of horse. If a significant interaction $(\mathrm{p}<0.10)$ of PRP line and group was found, then paired group differences were examined separately for each PRP line. An unstructured covariance structure was used in all repeated measures models. Student's t-tests were performed to test the hypothesis that change from control values were significantly different than 0 , which indicated a significant difference from control. All hypothesis tests were 2 -sided and the significance level was $\alpha=0.05$. Tukey's test was used to adjust for multiple paired comparisons.

\section{Author Contributions}

LB, JM, and JP conceived and designed experiments. LB, MT, JM performed experiments. RG assisted with experimental design and data interpretation. LB and JP wrote the manuscript.

\section{Conflicts of Interest}

The authors declare no conflict of interest.

\section{References}

1. Rossdale PD, Hopes R, Digby NJ, offord K (1985) Epidemiological study of wastage among racehorses 1982 and 1983. Vet Rec 116: 66-69.

2. Neundorf RH, Lowerison MB, Cruz AM, Thomason JJ, McEwen BJ, et al. (2010) Determination of the prevalence and severity of metacarpophalangeal joint osteoarthritis in Thoroughbred racehorses via quantitative macroscopic evaluation. Am J Vet Res 71: 1284-1293.

3. Jones MR, Ehrhardt KP, Ripoll JG, Sharma B, Padnos IW, et al. (2016) Pain in the Elderly. Curr Pain Headache Rep 20: 23.

4. McIlwraith CW, Frisbie DD, Kawcak CE (2012) The horse as a model of naturally occurring osteoarthritis. Bone Joint Res 1: 297-309.

5. Lampropoulou-Adamidou K, Lelovas P, Karadimas EV, Liakou C, Triantafillopoulos IK, et al. (2014) Useful animal models for the research of osteoarthritis. Eur J Orthop Surg Traumatol 24: 263-271.

6. Bendele AM (2001) Animal models of osteoarthritis. J Musculoskelet Neuronal Interact 1: 363-376.

7. Malfait AM, Little CB (2015) On the predictive utility of animal models of osteoarthritis. Arthritis Res Ther 17: 225.

8. Goodrich LR, Nixon AJ (2006) Medical treatment of osteoarthritis in the horse - a review. Vet J 171: 51-69.

9. Smyth NA, Murawski CD, Fortier LA, Cole BJ, Kennedy JG (2013) Platelet-rich plasma in the pathologic processes of cartilage: review of basic science evidence. Arthroscopy 29: 1399-1409. 
10. Reissis D, Tang QO, Cooper NC, Carasco CF, Gamie Z, et al. (2016) Current clinical evidence for the use of mesenchymal stem cells in articular cartilage repair. Expert Opin Biol Ther 16: 535-557.

11. Mohammadi S, Nikbakht M, Malek Mohammadi A, Zahed Panah M, Ostadali MR, et al. (2016) Human Platelet Lysate as a Xeno Free Alternative of Fetal Bovine Serum for the In Vitro Expansion of Human Mesenchymal Stromal Cells. Int J Hematol Oncol Stem Cell Res 10: 161-171.

12. Chiang ER, Ma HL, Wang JP, Liu CL, Chen TH, et al. (2016) Allogeneic Mesenchymal Stem Cells in Combination with Hyaluronic Acid for the Treatment of Osteoarthritis in Rabbits. PLoS One 11: 0149835.

13. Ozeki N, Muneta T, Koga H, Nakagawa Y, Mizuno M, Tsuji K, et al (2016) Not single but periodic injections of synovial mesenchymal stem cells maintain viable cells in knees and inhibit osteoarthritis progression in rats. Osteoarthritis Cartilage 24: 11061-1070.

14. de Windt TS, Vonk LA, Slaper-Cortenbach IC, van den Broek MP, Nizak R, et al. (2017) Allogeneic Mesenchymal Stem Cells Stimulate Cartilage Regeneration and Are Safe for Single-Stage Cartilage Repair in Humans upon Mixture with Recycled Autologous Chondrons. Stem Cells 35: 256264.

15. Wu CC, Sheu SY, Hsu LH, Yang KC, Tseng CC, et al. (2016) Intra-articular Injection of platelet-rich fibrin releasates in combination with bone marrow-derived mesenchymal stem cells in the treatment of articular cartilage defects: An in vivo study in rabbits. J Biomed Mater Res B Appl Biomater. 10: 1536-1543.

16. Sekiya I, Muneta T, Horie M, Koga H (2015) Arthroscopic Transplantation of Synovial Stem Cells Improves Clinical Outcomes in Knees With Cartilage Defects. Clin Orthop Relat Res 473: 2316-2326.

17. Carrade DD, Owens SD, Galuppo LD, Vidal MA, Ferraro GL, et al. (2011) Clinicopathologic findings following intra-articular injection of autologous and allogeneic placentally derived equine mesenchymal stem cells in horses. Cytotherapy 13: 419-430.

18. Keyser KA, Beagles KE, Kiem HP (2007) Comparison of mesenchymal stem cells from different tissues to suppress T-cell activation. Cell Transplant 16: 555-562.

19. Krampera M, Glennie S, Dyson J, Scott D, Laylor R, et al. (2003) Bone marrow mesenchymal stem cells inhibit the response of naive and memory antigen-specific T cells to their cognate peptide. Blood 101: 3722-3729.

20. Li H, Guo Z, Zhu H, Li XS, Jiang X, et al. (2010) Transplanted mesenchymal stem cells can inhibit the three developmental stages of murine acute graft-versus-host disease. In Vivo 24: 659-666.

21. Sudres M, Norol F, Trenado A, Grégoire S, Charlotte F, et al. (2006) Bone marrow mesenchymal stem cells suppress lymphocyte proliferation in vitro but fail to prevent graft-versus-host disease in mice. J Immunol 176 : 7761-7767.

22. Ferris DJ, Frisbie DD, Kisiday JD, McIlwraith CW, Hague BA, et al. (2014) Clinical outcome after intra-articular administration of bone marrow derived mesenchymal stem cells in 33 horses with stifle injury. Vet Surg 43: 255-265.

23. McIlwraith CW, Frisbie DD, Rodkey WG, Kisiday JD, Werpy NM, et al (2011) Evaluation of intra-articular mesenchymal stem cells to augment healing of microfractured chondral defects. Arthroscopy 27: 1552-1561.

24. Kim YS, Kwon OR, Choi YJ, Suh DS, Heo DB, et al. (2015) Comparative Matched-Pair Analysis of the Injection Versus Implantation of Mesenchymal Stem Cells for Knee Osteoarthritis. Am J Sports Med 43: 2738-2746.

25. Shimojo AA, Perez AG, Galdames SE, Brissac IC, Santana MH (2015) Performance of PRP associated with porous chitosan as a composite scaffold for regenerative medicine. Scientific World Journal 2015: 396131.

26. Xie A, Nie L, Shen G, Cui Z, Xu P, et al. (2014) The application of autologous platelet-rich plasma gel in cartilage regeneration. Mol Med Rep 10: 1642-1648.
27. Mishra A, Tummala P, King A, Lee B, Kraus M, et al. (2009) Buffered platelet-rich plasma enhances mesenchymal stem cell proliferation and chondrogenic differentiation. Tissue Eng Part C Methods 15: 431-435.

28. Tang HC, Chen WC, Chiang CW, Chen LY, Chang YC, et al. (2015) Differentiation Effects of Platelet-Rich Plasma Concentrations on Synovial Fluid Mesenchymal Stem Cells from Pigs Cultivated in Alginate Complex Hydrogel. Int J Mol Sci 16: 18507-18521.

29. Kreuz PC, Krüger JP, Metzlaff S, Freymann U, Endres M, et al. (2015) Platelet-Rich Plasma Preparation Types Show Impact on Chondrogenic Differentiation, Migration, and Proliferation of Human Subchondral Mesenchymal Progenitor Cells. Arthroscopy 31: 1951-1961.

30. Yang H, Li ZT, Lin R, Fan ZP, Huang F, et al. (2016) [Sclerodermatous chronic graft-versus-host disease after hematopoietic stem cell transplantation: incidence, clinical characteristics and risk factors]. Nan Fang Yi Ke Da Xue Xue Bao 36: 807-813.

31. van Buul GM, Koevoet WL, Kops N, Bos PK, Verhaar JA, et al. (2011) Platelet-rich plasma releasate inhibits inflammatory processes in osteoarthritic chondrocytes. Am J Sports Med: 39: 2362-2370.

32. Pereira RC, Scaranari M, Benelli R, Strada P, Reis RL, et al. (2013) Dual effect of platelet lysate on human articular cartilage: a maintenance of chondrogenic potential and a transient proinflammatory activity followed by an inflammation resolution. Tissue Eng Part A 19: 1476-1488.

33. Anitua E, Sánchez M, Nurden AT, Zalduendo MM, de la Fuente M, et al. (2007) Platelet-released growth factors enhance the secretion of hyaluronic acid and induce hepatocyte growth factor production by synovial fibroblasts from arthritic patients. Rheumatology (Oxford) 46: 769-772.

34. Sundman EA, Cole BJ, Karas V, Della Valle C, Tetreault MW, et al. The anti-inflammatory and matrix restorative mechanisms of platelet-rich plasma in osteoarthritis. Am J Sports Med 42: 35-41.

35. Broeckx S, Zimmerman M, Crocetti S, Suls M, Mariën T, et al. (2014) Regenerative therapies for equine degenerative joint disease: a preliminary study. PLoS One 9: 85917.

36. Yun S, Ku SK, Kwon YS (2016) Adipose-derived mesenchymal stem cells and platelet-rich plasma synergistically ameliorate the surgical-induced osteoarthritis in Beagle dogs. Journal of Orthopaedic Surgery and Research 11 .

37. Milano G, Sanna Passino E, Deriu L, Careddu G, Manunta L, et al. (2010) The The effect of platelet rich plasma combined with microfractures on the treatment of chondral defects: an experimental study in a sheep model. Osteoarthritis Cartilage 18: 971-980.

38. do Amaral RJ, da Silva NP, Haddad NF, Lopes LS, Ferreira FD, et al. (2016) Platelet-Rich Plasma Obtained with Different Anticoagulants and Their Effect on Platelet Numbers and Mesenchymal Stromal Cells Behavior In Vitro. Stem Cells Int 2016: 7414036.

39. do Amaral RJ, Matsiko A, Tomazette MR, Rocha WK, Cordeiro-Spinetti E, et al. (2015) Platelet-rich plasma releasate differently stimulates cellular commitment toward the chondrogenic lineage according to concentration. J Tissue Eng 6.

40. Amable PR, Teixeira MV, Carias RB, Granjeiro JM, Borojevic R (2014) Mesenchymal stromal cell proliferation, gene expression and protein production in human platelet-rich plasma-supplemented media. PloS one 9: 104662 .

41. Atashi F, Jaconi ME, Pittet-Cuénod B, Modarressi A (2015) Autologous platelet-rich plasma: a biological supplement to enhance adipose-derived mesenchymal stem cell expansion. Tissue Eng Part C Methods 21: 253262.

42. Wuchter P, Vetter M, Saffrich R, Diehlmann A, Bieback K, et al. (2016) Evaluation of GMP-compliant culture media for in vitro expansion of human bone marrow mesenchymal stromal cells. Exp Hematol 44: 508-518. 
43. Russell KA, Koch TG (2016) Equine platelet lysate as an alternative to fetal bovine serum in equine mesenchymal stromal cell culture - too much of a good thing? Equine Vet J 48: 261-264.

44. Pak J, Chang JJ, Lee JH, Lee SH (2013) Safety reporting on implantation of autologous adipose tissue-derived stem cells with platelet-rich plasma into human articular joints. BMC Musculoskelet Disord 14: 337.

45. Zhu Y, Yuan M, Meng HY, Wang AY, Guo QY, et al. (2013) Basic science and clinical application of platelet-rich plasma for cartilage defects and osteoarthritis: a review. Osteoarthritis Cartilage 21: 1627-1637.

46. Van Pham P, Bui KH, Ngo DQ, Vu NB, Truong NH, et al. (2013) Activated platelet-rich plasma improves adipose-derived stem cell transplantation efficiency in injured articular cartilage. Stem Cell Res Ther 4: 91

47. Creeper F, Ivanovski S (2012) Effect of autologous and allogenic platelet-rich plasma on human gingival fibroblast function. Oral Dis 18: 494500 .

48. Li Y, Lin F (2012) Mesenchymal stem cells are injured by complement after their contact with serum. Blood 120: 3436-3443.

49. Del Bue M, Ricco S, Conti V, Merli E, Ramoni R, et al. (2007) Platelet lysate promotes in vitro proliferation of equine mesenchymal stem cells and tenocytes. Vet Res Commun 1: 289-292.

50. Del Bue M, Ricco S, Ramoni R, Conti V, Gnudi G, et al. (2008) Equine adipose-tissue derived mesenchymal stem cells and platelet concentrates: their association in vitro and in vivo. Vet Res Commun 1: 51-55.

51. Kol A, Walker NJ, Galuppo LD, Clark KC, Buerchler S, et al. (2013) Autologous point-of-care cellular therapies variably induce equine mesenchymal stem cell migration, proliferation and cytokine expression. Equine Vet J 45: 193-198.
52. Seo JP, Tsuzuki N, Haneda S, Yamada K, Furuoka H, et al. (2013) Comparison of allogeneic platelet lysate and fetal bovine serum for in vitro expansion of equine bone marrow-derived mesenchymal stem cells. Res Vet Sci 95: 693-698

53. Garvican ER, Cree S, Bull L, Smith RK, Dudhia J (2014) Viability of equine mesenchymal stem cells during transport and implantation. Stem Cell Res Ther 5: 94.

54. Man Y, Wang P, Guo Y, Xiang L, Yang Y, et al. (2012) Angiogenic and osteogenic potential of platelet-rich plasma and adipose-derived stem cell laden alginate microspheres. Biomaterials 33: 8802-8811.

55. Josh F, Kobe K, Tobita M, Tanaka R, Suzuki K, et al. (2012) Accelerated and Safe Proliferation of Human Adipose-derived Stem Cells in Medium Supplemented with Human Serum. J Nippon Med Sch 79: 444-452.

56. Kol A, Walker NJ, Galuppo LD, Clark KC, Buerchler S, et al. (2012) Autologous point-of-care cellular therapies variably induce equine mesenchymal stem cell migration, proliferation and cytokine expression. Equine Vet J 45: 193-198.

57. Kruger JP, Hondke S, Endres M, Pruss A, Siclari A, et al. (2012) Human platelet-rich plasma stimulates migration and chondrogenic differentiation of human subchondral progenitor cells. J Orthop Res 30: 845-852.

58. Xie X, Wang Y, Zhao C, Guo S, Liu S, et al. (2012) Comparative evaluation of MSCs from bone marrow and adipose tissue seeded in PRP-derived scaffold for cartilage regeneration. Biomaterials 33: 7008-7018.

59. Goodrich LR, Chen AC, Werpy NM, Williams AA, Kisiday JD, et al. (2016) Addition of Mesenchymal Stem Cells to Autologous Platelet-Enhanced Fibrin Scaffolds in Chondral Defects: Does It Enhance Repair? J Bone Joint Surg Am 6: 23-34. 